See discussions, stats, and author profiles for this publication at: https://www.researchgate.net/publication/332472143

\title{
Contracting in the Smart Era: The Implications of Blockchain and Decentralized Autonomous Organizations for Contracting and Corporate Governance
}

Article in Academy of Management Perspectives · April 2019

DOI: $10.5465 / \mathrm{amp} .2018 .0066$

CITATIONS

8

4 authors, including:

Alex Murray

University of Oregon

9 PUBLICATIONS 19 CITATIONS

SEE PROFILE

Matt Josefy

Indiana University Bloomington

18 PUBLICATIONS 293 CITATIONS

SEE PROFILE

Some of the authors of this publication are also working on these related projects:
READS

1,191

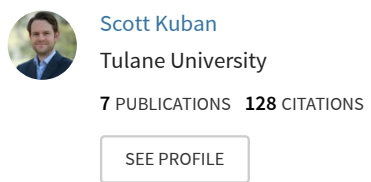

Ill things great and small: Organizational size, boundaries of the firm and a changing environment View project 


\title{
CONTRACTING IN THE SMART ERA: THE IMPLICATIONS OF BLOCKCHAIN AND DECENTRALIZED AUTONOMOUS ORGANIZATIONS FOR CONTRACTING AND CORPORATE GOVERNANCE
}

\author{
Alex Murray \\ University of Oregon \\ amm16@uoregon.edu \\ Scott Kuban \\ Tulane University \\ skuban@tulane.edu

\begin{abstract}
Matthew Josefy
mjosefy@iu.edu
\end{abstract} \\ Indiana University \\ Jon Anderson \\ Indiana University \\ janderson@cmtam.com
}

\section{Accepted at Academy of Management Perspectives}

We gratefully acknowledge feedback received at the 2018 Academy of Management Specialized Conference at the University of Surrey. We also appreciate the constructive comments received from two anonymous reviewers and the direction provided from the editor, Phillip Phan, which substantially improved the quality of this work. All authors contributed equally and are listed in reverse alphabetical order. 


\title{
CONTRACTING IN THE SMART ERA: THE IMPLICATIONS OF BLOCKCHAIN AND DECENTRALIZED AUTONOMOUS ORGANIZATIONS FOR CONTRACTING AND CORPORATE GOVERNANCE
}

\begin{abstract}
This paper explores blockchain technology's potential to alter contracting both in the market and within organizations. We identify and discuss how blockchain reduces certain types of transaction costs while introducing additional costs that have not been present in traditional contracts. Blockchain technology also presents a new method to mitigate or avoid certain types of agency costs that stem from contracting with agents inside the firm. Through this theoretical discussion, our paper proposes several avenues for future research on how blockchain may alter contracting and corporate governance.
\end{abstract}

Keywords: blockchain technology, contracting, corporate governance, transaction costs, agency costs, smart contracts, decentralized autonomous organizations (DAOs) 


\section{INTRODUCTION}

Scholars have theorized that firms exist and grow larger, in part, to avoid and manage contracting hazards that exist when conducting market transactions (Josefy, Kuban, Hitt \& Ireland, 2015; Mayer \& Salomon, 2006; Williamson, 1981; Zenger, Felin \& Bigelow, 2011). Certain firms may even develop a superior capability for designing or managing contracts with external parties (Argyres \& Mayer, 2007; Mayer \& Salomon, 2006; Weber \& Mayer, 2011). Within the firm itself, where ownership and management are often separated to coordinate complex tasks, several costs also arise from contracting with the agents who manage the firm (Berle \& Means, 1932; Jensen \& Meckling, 1976). Firm success is thus tied to a combination of internal and external contracts. As such, significant—-though generally separate_-bodies of work have addressed how firms manage contracts with external parties as well as manage the agency costs that arise from contracting with their own managers. However, technological advancements are providing new methods of contracting that warrant scholarly attention as they may alter longstanding assumptions and previous findings in both areas of scholarly inquiry.

This paper examines the potential implications of one specific technological advancement, the blockchain-enabled distributed ledger, or, put simply, blockchain technology. While many people are becoming aware of blockchain-enabled cryptocurrencies such as Bitcoin, they tend to be less familiar with the role of blockchain technology in facilitating person-toperson market transactions or its extensive uses beyond just cryptocurrency transactions. Defined as immutable chains that ensure data transparency, blockchain technology enables preprogrammed algorithms and protocols to monitor connected inputs automatically, respond to 
changes, enforce rules, and trigger responses ${ }^{1}$. More complicated preprogrammed algorithms are commonly referred to as smart contracts and are discussed in more detail later in this paper.

Proponents suggest that blockchain technology has the capabilities to disrupt several industries by removing intermediaries and increasing market efficiencies (e.g., Michelman, 2017; Tapscott \& Tapscott, 2017). Further, blockchain technology is increasingly being used by established firms, and has even enabled an entirely new organizational form-the decentralized autonomous organization (DAO) — managed entirely through protocols that are encoded and enforced via smart contracts rather than human managers. In combination, these developments indicate that blockchain technology is beginning to impact the way certain contracts are established and executed, reduce the costs associated with agent-managers, and even fundamentally alter the way some firms are governed. As a community of management scholars, we would benefit from understanding the implications of blockchain technology for contracting in general and on corporate governance practices in particular (Seidel, $2018 \mathrm{~A}$ ).

The advent of blockchain technology requires the development of additional theory for two reasons: one in the market and the other within the firm. In the market, self-executing smart contracts can govern certain transactions. Within the firm, blockchain provides a technological solution to mitigate opportunities for managers' mistakes or malfeasance, and, in the extreme case of DAOs, eliminate the need for managers altogether. In combination, these new possibilities have three potential theory-based implications that we address in this paper. First, while Coase and Williamson relied on the assumption that complex and uncertain transactions need to be orchestrated within a firm's boundaries, organizations may now have a new option to

\footnotetext{
${ }^{1}$ Connected inputs and triggered responses can be completely digital in nature (e.g., stock price feeds) or any of the myriad of intranet/internet connected devices commonly referred to as the Internet of Things.
} 
conduct complex and uncertain transactions via contracts outside the organization. Second, blockchain technology may reduce or eliminate certain principal-agent costs that arise from contracting with managers within the firm. Finally, increased reliance on blockchain technology may introduce new costs for firms that have received little consideration in prior theorizing, yet affect a firm's decisions regarding whether to conduct particular transactions within or outside the firm's boundaries.

In this paper, we first broadly introduce blockchain technology and discuss key concepts and applications for the technology. We next briefly summarize the existing literature on contracting in the market and agency costs that arise from contracting within the firm. We then consider blockchain's impact on contracting by introducing new potential benefits as well as new costs for firms. Finally, we discuss the implications for contracting in the market using blockchain as well as blockchain's potential to mitigate five types of agency costs that firms have historically faced. We aim to catalyze an expansive line of future inquiry on the impact of blockchain technology on contracting both in the market and within firms.

\section{BLOCKCHAIN TECHNOLOGY}

In 2008, the term blockchain emerged to describe the foundational technology of Bitcoin $^{2}$, a "peer-to-peer electronic cash system" developed by the anonymous Satoshi Nakamoto (Nakamoto, 2008). During the following decade, several groups became increasingly interested in the promise of blockchain technology, including programmers and early adopters intrigued by the technology's potential, libertarians yearning for a decentralized society, and

\footnotetext{
${ }^{2}$ The term "Bitcoin" is capitalized when referencing the protocol or network (e.g., Bitcoin was founded in 2009); and the term "bitcoin" is not capitalized when referencing the unit of currency (e.g., she purchased 10 bitcoin).
} 
founders of financial-tech startups seeking to disrupt the (inefficient and highly-intermediated) banking industry. But it was not until more recently that blockchain moved beyond these niches. In the final months of 2017, the term blockchain entered the mainstream vernacular. The rapid appreciation in the price of bitcoin was highlighted further as newspapers and magazines profiled "Bitcoin millionaires and billionaires" (Ambler, 2018; Bowles, 2018; Mahdawi, 2018). Meanwhile, experts and media alike debated blockchain's potential to disrupt established industries such as banking and commodities trading, or even usher in a new era of decentralization altogether (Bogost, 2017). New start-ups pursued funding by issuing tokens (i.e., cryptocurrencies) based on blockchain while interest and trading in cryptocurrencies (e.g., Bitcoin, Ethereum, and Litecoin) skyrocketed worldwide. Consumers' fear of missing out contributed to a rapid rise in the value of cryptocurrencies (Guadiano, 2018), which was then followed by substantial pricing corrections. Regulators, including the U.S. Securities and Exchange Commission, have begun to actively consider what role, if any, they should play in overseeing the markets created by this new technology (Roberts, 2018).

Blockchain technology consists of a distributed ledger that processes and chronologically records transactions on a network of computers to form an "immutable chain" through protocols and network consensus without relying on centralized intermediaries. Essentially, blockchain technology is a decentralized database that operates by recording transactions (as well as digitized smart contracts and other forms of information) on a series of distributed computers. These secure records are then available and transparent to a broader network of participants (Mougayar, 2016). This new technology allows validation of direct peer-to-peer transactions of digital assets without the presence of established and reputable intermediaries to ensure trust between exchange partners. Perhaps the most commonly-known use case of blockchain is the 
cryptocurrency Bitcoin. Bitcoin is an Internet-enabled digital money system that is not restricted or defined by a geographic region or geopolitical entity. However, Bitcoin is a comparatively limited blockchain focused on a single use case: a distributed currency that enables peer-to-peer financial transactions. Other blockchains have been set up to allow for much greater flexibility and therefore a wide variety of use cases.

As an illustrative example of how a blockchain-enabled transaction is different than a traditional one, we compare a ride-sharing transaction using Uber with a hypothetical blockchain-based competitor (see Seidel, 2018 B). Both transactions begin with a human request via a local smartphone application that serves as the user interface to the platform. However, while an Uber-controlled server farm handles user validation, payment processing (with help from an additional financial intermediary), and execution (e.g., assigning a driver); the blockchain version is broadcast to a widely distributed network of computers, commonly referred to as miners ${ }^{3}$. These dispersed mining computers serve two functions: (1) to validate and process current transactions by reaching consensus and (2) to maintain and ensure the security of the blockchain record of all prior transactions. Miners are compensated for the use of their computers with digital tokens of value built into the blockchain system. The ride-sharing firm, therefore, pays for processing each transaction (based on computational usage) with digital tokens it has purchased or otherwise acquired prior to the transaction. Processing this example ride-sharing transaction also includes executing commands to dispatch a nearby driver to the user's location and transferring payment after drop-off ${ }^{4}$. Finally, upon completion, the

\footnotetext{
${ }^{3}$ The term "miner" comes from an early conceptualization that computers are "mining" for digital tokens.

${ }^{4}$ While the distributed network of blockchain computers can perform any computer processing function, the cost of using miners' computers is generally higher relative to other computers (see Senta, 2017 for approximate transaction
} 
transaction is recorded with other recent transactions to create a new block of data in the distributed ledger that is then added to the existing chain of data blocks, or the blockchain. The distributed structure and computationally-intensive nature of the blockchain make it, for most intents and purposes, impossible to alter the ledger by any party involved in the transaction or by nefarious third parties.

As previously mentioned, a blockchain transaction can encompass a complex array of inputs from and outputs to other computer systems and/or any connected device in the physical world. While the underlying blockchain network setup can limit a transaction's complexity, as is the case with the Bitcoin blockchain, more recent blockchains have been set up to allow for "Turing complete" programmability. Most notably, the Ethereum blockchain was one of the first to implement a fully programmable setup and thereby popularize the term smart contract to describe more complex, automated blockchain transactions (Buterin, 2013). Smart contracts enable multiple parties who do not know (or trust) one another to engage in exchanges of value when certain conditions are satisfied. Essentially, smart contracts are small programs that automatically execute when pre-specified conditions (i.e., rules) in the protocols are satisfied.

Given their automaticity, smart contracts do not depend on third-party intermediaries or human agency for their execution. Instead, smart contracts rely on a variety of trusted data feeds,

costs). So, while not necessary, it is likely that the smart contract would be programmed to offload tasks that do not require blockchain's advantages (e.g., determining the closest driver) to a non-blockchain server or the smartphone application for the cost savings.

${ }^{5} \mathrm{~A}$ computer or a programming language is said to be Turing complete if it can implement a Turing machine. A Turing machine is a mathematical model of computation that can, in principle, perform any calculation that any other programmable computer can perform (see De Mol, 2018 for a more detailed explanation). 
or oracles, to securely transfer external data that corresponds with preprogrammed, algorithmic protocols stored on a blockchain. Examples of "oraclized" data inputs include fully autonomous sensors (e.g., rain gauges, digital thermometers), online inputs (e.g., website traffic, stock price changes, sporting outcomes), and real-world human decisions (e.g., Supreme Court rulings, trade agreements). Any of these inputs can trigger the preprogrammed protocols stored on a blockchain to automatically carry out a range of actions such as executing financial transactions or sending commands to smart devices. With the ever-increasing number of Internet-connected devices $^{6}$, the abilities of smart contracts are continuing to expand (Christidis \& Devetsikiotis, 2016). It is important to note that oracles, while more automated and potentially devoid of human discretion, are not infallible. We discuss this oracle problem further in a later section. We next review the relevant literature. First, we discuss transaction cost economics (TCE) and the theory of the firm. Then, we turn to agency theory and its associated agency costs.

\section{RELEVANT PRIOR LITERATURE}

\section{Contracting, TCE, and the Theory of the Firm}

Transaction costs are an important consideration for understanding why some transactions occur in the general market, while others are conducted within the boundaries of a firm (Coase, 1937; Williamson, 1981). Scholars have demonstrated in many settings how factors such as asset specificity and uncertainty can affect transaction costs and, in turn, what is the optimal mode — market or firm — for carrying out various transactions (Williamson, 1979). Further, this perspective has highlighted the critical role of contracts in governing economic exchange - to the point that the firm has been characterized as a "nexus of contracts" (e.g.,

\footnotetext{
${ }^{6}$ Bain predicts the Internet of Things (IoT) market will expand from $\$ 235$ billion in 2017 to $\$ 520$ billion by 2021 (Columbus, 2018).
} 
Fama, 1980). Indeed, the modern, hierarchical corporate form emerged to manage transactions and capital efficiently. However, hierarchy also creates its own inefficiencies, notably through the complexity of task coordination (Simon, 1977; Thompson, 1967). Since coordination increases the importance of owners employing professional managers, principal-agent conflicts emerge that then create a significant cost of contracting within the firm. This is discussed in detail in a later section.

Contracting hazards generally push transactions into firm hierarchies, while the absence of such hazards — or the ability to mitigate and monitor those hazards — allows firms to instead conduct such transactions in the open market (Mayer \& Salomon, 2006). Contracts vary significantly in their scope, form, description of terms and conditions, and complexity (Schepker, Oh, Martynov \& Poppo, 2014). Certain firms develop a superior ability to design contracts to include provisions that best address particular transaction attributes, in part by drawing on knowledge held by various parties within the firm (Argyres \& Mayer, 2007). Even when contracts are well drawn up, their use and implementation significantly affect and are affected by relationships between the transacting parties (Weber \& Mayer, 2011). In this vein, the execution of frequently-occurring contractual agreements is often routinized within a firm (Grant, 1996). Such routinized processes represent repetitive and recognizable patterns of interdependent actions between multiple actors that impact a firm's behavior (Cyert \& March, 1963; Feldman, 2000; Feldman \& Pentland, 2003; Howard-Grenville, 2005; Nelson \& Winter, 1982; Parmigiani \& Howard-Grenville, 2011). These routines help to minimize contracting hazards and costs. Because contracting is such a pivotal activity within firms, any means to improve it has the potential to have considerable bearing on whether transactions occur in the market or within a firm's boundaries (Josefy et al., 2015; Zenger et al., 2011). It is for this reason that blockchain, 
as an enabler of smart contracts, has important ramifications for theory, as it could fundamentally alter whether certain frictions or costs are or are not present in transactions. Smart contracts fundamentally represent a routinization of certain processes_-reducing them down to articulated conditions, monitoring for those conditions, and executing on those conditions ${ }^{7}$. While the implications of smart contracts are further discussed in a later section, it is valuable to consider smart contracts from the perspective of how they alter transaction costs and, in turn, how they affect firm boundaries. We now consider a specific type of contracting cost that exists for firms: agency costs.

\section{Agency Theory and Agency Costs}

Despite the many benefits of coordinating transactions within a firm's boundaries, corporations also create the possibility for interests to diverge between owners - principals - and those managing a firm's activities_-agents (Daily, Dalton, \& Cannella, 2003; Daily, Dalton, \& Rajagopalan, 2003; Dalton, Hitt, Certo, \& Dalton, 2007; Jensen \& Meckling, 1976). Agency theory posits that the interests of owners and managerial agents routinely diverge and that managers can use the inherent information asymmetry that arises from their positioning within a firm to pursue personal rather than profit-maximizing goals that align with owners' interests (Dalton et al., 2007; Fama \& Jensen, 1983; Jensen \& Meckling, 1976). This informational asymmetry is exacerbated in publicly traded firms, as shareholders possess fewer legal rights relative to owners because those rights ${ }^{8}$ reside instead with a firm's board of directors (Stout, 2002). These conflicts of interest, and firms' actions to mitigate them, provide the basis for a

\footnotetext{
${ }^{7}$ We are grateful to an anonymous reviewer for this observation and for suggesting this wording.

${ }^{8}$ Rights such as control over the corporation's assets, disbursements from earnings, or access to accounting information beyond SEC requirements.
} 
long line of theoretical and empirical inquiry on principal-agent relationships (Eisenhardt, 1989). While prior studies identify several tactics that firms can use to mitigate principal-agent conflicts (e.g., Conlon \& Parks, 1990; Kaplan \& Stromberg, 2001), each of these tactics incur agency costs to a firm. Thus, agency costs encompass both losses from self-serving agent behavior and the costs incurred by the firm in trying to prevent such behavior (Jensen \& Meckling, 1976).

Prior research has thus focused on mitigating agency costs arising from agent monitoring, self-serving agent behaviors and, when possible, better aligning the interests of managers with owners (e.g., Carpenter \& Sanders, 2002; Tosi, Katz \& Gomez-Mejia, 1997). We identify and discuss five types of agency costs from prior literature. First, one agency cost is the monitoring of agent motivations to detect and deter self-serving behavior (Ang, Cole, \& Lin, 2000; Daily \& Dalton, 1995; Dalton et al., 2007). The second cost arises from monitoring a firm's operations to reduce the informational advantage that agent-managers have over owners and shareholders. For instance, owners can monitor firm operations by hiring auditors to verify the veracity of managerial claims. The third type of agency cost is excessive expenses such as managerial perks (Ang et al., 2000; Jensen \& Meckling, 1976). Fourth, substantial funds are spent on "interest alignment" of the agent-manager to reduce, if not eliminate, the divergence from owners' interests (Nyberg, Fulmer, Gerhart, \& Carpenter, 2010). Finally, unrealized profits from "suboptimal" management (i.e., any non-profit-maximizing decisions or behaviors carried out by agent-managers) are also a type of agency cost (Hoskisson, Wan, Yiu, \& Hitt, 1999; Hoskisson $\&$ Turk, 1990; Tosi \& Gomez-Mejia, 1989). In combination, these five costs represent the value that owners forego from contracting with managers to run the company on their behalf. 
In the following section, we discuss both the promise of blockchain technology and the costs incurred by blockchain technology's adoption. Then we examine the overall implications of blockchain for contracting in the market and within the firm.

\section{HOW BLOCKCHAIN ALTERS CONTRACTING}

As firms have long sought to optimize contracting, the costs associated with contracting have played a key role in theorizing about the existence and boundaries of firms (e.g., Coase, 1937; Williamson, 1985). In this section, we consider how blockchain technology may alter contracting both in the market and within the firm and thereby affect our understanding of firms that has long been tied to prior assumptions about the relative costs of both types of contracting. First, we examine the promise of blockchain technology to change some of the prior assumptions regarding contracting. Next, we consider new costs introduced with blockchain technology adoption that contribute to the frictions of using "smart contracts." Then, we discuss the implications of blockchain for contracting in the market. Finally, we examine five agency costs associated with agent-managers within firms and the implications of blockchain for contracting within the firm.

\section{The Promise of Blockchain Technology Adoption}

The promise of blockchain technology is far-reaching. First, it has spurred new ventures with business models built around blockchain's technological advantages. Second, established firms are incorporating it to improve their operations. Finally, it has led to the creation of a new organizational form, DAOs, which pose a foundational threat to our classic scholarly understanding of the firm.

Since Ethereum's inception, many start-ups have developed decentralized applications using the Ethereum blockchain's smart contract capabilities. One such example is Slock.it, a 
start-up that uses blockchain-enabled smart locks to allow completely automated renting in the sharing economy. For instance, Slock.it's system allows instant (for the user) and authenticated (for the owner) access to an Airbnb apartment via smart deadbolts after taking a verified payment. Padlock-style smart locks further extend Slock.it's system to include many other rental objects such as bicycles and vehicles. Another example is Uport, an application that provides users with a blockchain alternative for proving one's identity independent from government entities (e.g., a driver's license) or centralized third-parties (e.g., "login with your Facebook account"). A third start-up, Provenance, is using the Ethereum blockchain to make opaque supply chains more transparent by creating a platform to trace the origin and history of various products.

While start-ups are creating entire business models around blockchain-enabled applications, established firms are also beginning to utilize blockchain-enabled smart contracts to improve aspects of their operations. For example, IBM recently partnered with Maersk to develop a platform (not unlike the previous Provenance example) to address opaque and inefficient global supply chains by providing "digital authenticity and immutability of digital documents" to all supply chain participants while reducing the need for inefficient third-party authenticators throughout the shipping process (White, 2018). Meanwhile, Northern Trust is using blockchain technology to simplify the administration and even automate much of the legal paperwork involved in complex private equity deals (Northern Trust, 2017). While the above examples are profound, the impact of smart contracts is expanding far beyond emergent platforms and established supply chains to impact the very way firms are managed.

Perhaps most interesting for management scholars, blockchain technology has enabled an entirely new organizational form: DAOs. As the most extreme case of a blockchain-enabled 
company, DAOs are organizations run entirely through protocols that are encoded and enforced via smart contracts. An early, and now infamous, example of a DAO was eponymously named "The DAO." This was an investor-directed venture capital fund without a hierarchical structure, centralized fund managers, or a board of directors. Instead, owner-investors possessed direct rights to submit investment proposals and vote on the allocation of The DAO's pooled capital. Investments were then executed based on smart contracts when certain pre-specified voting criteria were met. The premise was simple, by taking control away from traditional fund managers and placing it in the hands of investor-owners, investors could realize the benefits of pooling their investment capital without worrying about fund managers misdirecting or wasting their funds. While The DAO had a promising start, raising over $\$ 150$ million during its digital token crowdsale in May $2016^{9}$, it shut down when a hacker exploited vulnerabilities in its code. However, like many early technologies, The DAO's failure provided opportunities for coders to learn from its flaws to then inform the development of more secure decentralized and autonomous organizations in the future. The DAO's founding team subsequently launched Charity DAO, a donor-led, blockchain-enabled "truly not-for-profit charity" with no employees or operating costs (Jentzsch, 2016 A).

Taken together, these developments indicate that blockchain technology may affect some of the prior assumptions regarding contracting in the market, the costs associated with agentmanagers within firms, and even fundamentally alter the way some firms are managed. However, adopting blockchain technology can introduce new costs for firms as well.

\footnotetext{
${ }^{9}$ By many standards, The DAO's ownership was also largely “democratized” as the top 100 investors held only 46 percent of the voting-right tokens (Chavez-Dreyfuss, 2016).
} 


\section{New Costs Arising from Blockchain Technology Adoption}

We suggest blockchain technology will introduce three new costs for firms to contend with when determining whether to transact via smart contracts. First, inflexibility costs arising from a lack of discretion in applying smart contracts' preprogrammed rules can introduce new contracting costs for firms. Second, oracle costs, relating to the "oracle problem," can lead to new costs when information intermediaries provide flawed or incorrect information that inappropriately triggers a smart contract's execution. Finally, security costs associated with ensuring the safety and reliability of the blockchain protocols and its data records can result in additional costs as well. We will now discuss each of these costs in further detail.

New costs arising from inflexibility. Since smart contracts execute without human intermediaries, inflexibility costs include those that result from removing human discretion in a contract's execution. In other words, these costs encompass those that a firm incurs when a contract is automatically executed based on the "letter of the law" as written into the contract rather than the contract's underlying "spirit." Since smart contracts are executed entirely from encoded if-then statements that are written at the outset of agreements between transacting parties, variance in a smart contract's enforcement is rigid and inflexible (Sklaroff, 2018). This inflexibility is particularly important to consider since all contracts inherently give rise to interpretative uncertainty since the contracting parties may have different views of the exchange (Weber \& Mayer, 2014). Thus, contracting partners often develop norms that govern their settlement of repeated contracts (Schepker et al., 2014). To reconcile potential disagreements arising from smart contract execution, firms are apt to incur costs for specialized arbitration services that specialize in smart contracting. 
In returning to the example of The DAO, the hack in June 2016 exploited a loophole in The DAO’s smart contracts to drain nearly $\$ 50$ million from its investment fund. Due to The DAO's fully decentralized and autonomous governance structure, no centralized agent-managers could make fast decisions to (attempt to) stop the hack in real time or even correct bugs in the code after the hack concluded. As such, the lack of centralized agent-managers ironically contributed to The DAO's inevitable demise. In addition to highlighting the inflexibility of smart contracts, this example also implicates the important role agent-managers play in making timely and adaptive decisions in the occurrence of rare events such as hacks or data breaches by putting systems and processes in place to prevent similar events from occurring in the future.

Though blockchain-enabled smart contracts are an effective means to eliminate agentmanagers in the case of DAOs, it remains unclear if and how established firms can employ blockchain technology to reduce agent-managers' control. One option for firms seeking to mitigate the costs of monitoring agent-managers is to democratize core operational decisions (e.g., investment decisions in an investment fund) using blockchain technology while maintaining agent-managers' ability to intervene when certain rare events occur. This would mitigate principal-owners concerns over manager-agents' motivations in most day-to-day circumstances while entrusting managers to intervene in more dire situations. Yet, if firms were to use blockchain in this way, it would be imperative for structures to be instituted that determine the conditions that enable an agent-manager's intervention. Another option is to provide managers with a "pause" function to halt a smart contract's automatic execution and thus provide ample time for consensus to be reached. While these are both options for firms to consider, providing managers with discretion to override or pause a smart contract's execution undermines 
some of the advantages of self-executing smart contracts. Further inquiry can assess the optimal structures firms can employ to mitigate agent-managers' control when using smart contracts.

New costs arising from the "oracle problem." Blockchain enthusiasts have suggested that blockchain eliminates intermediaries (Gupta, 2017). However, in their place, it is worth noting that many smart contracts must still consult with one or more third-party information sources, also known as oracles. Similarly, in Greek mythology, the Delphic Oracle could be consulted by individuals to obtain answers to otherwise confounding questions and "Greek and barbarian alike consulted it" (Dempsey \& Conway, 1918: 38). Yet, while the Delphic Oracle offered a singular source for authoritative prophecies, firms face the more challenging task of identifying appropriate oracles given the vast number of available options with varying degrees of accuracy, reliability, security, speed, and price. As such, the oracle problem can result in significant costs.

Firms may experience difficulty in selecting appropriate third-party information intermediaries to provide inputs for execution when writing smart contracts. For instance, if a smart contract guarantees that a supplier will receive a payment if oil prices rise, it is necessary for the firm to identify a data source for oil price information that is adequately accurate, reliable, and secure from external tampering at a cost that is at least competitive with alternative (traditional) contracting costs. Depending on a contract's terms, the speed of data updates could also be a factor. Inaccurate oracle data could lead to further costs post-contract execution. This makes selection crucial. For example, if the selected oracle for oil prices provides inaccurate or delayed information, the firm will incur costs to (attempt to) remedy the incorrectly executed transaction. However, it can be difficult and/or costly to determine ex-ante the accuracy of a data 
source without a historical record accessible for review ${ }^{10}$. A lack of such records could give rise to "reputations" for data sources such as prior customer reviews, as is common in the sharing economy; or a third-party rating system similar to a credit rating. The latter, ironically, would shift the "source of trust" again to a centralized third party. This would likely also lead firms to incur costs for the services of "oracle rating agencies" which evaluate potential oracles for a fee.

It is therefore important to note that oracles can vary in the degree to which human discretion is reflected in the information they provide. For example, a barometer reading provides an objective measurement ${ }^{11}$. As such, the primary concern in using this data source as an input for a smart contract is the timeliness of the information's transfer. Periodicals such as The Wall Street Journal could also serve as an oracle for a smart contract. Given the role of humans in reporting for such publications, articles can contain factual errors due to misreporting that result in subsequent retractions or amendments to the original article. Misreporting could therefore negatively impact a firm if it leads to a contract's incorrect execution. Firms will consequently incur costs to identify the most appropriate and reliable sources of data to ensure contracts execute appropriately; thereby avoiding subsequent costs from misexecution. In this way, the oracle problem results in costs both when writing complete contracts (i.e., pre-contract execution) and in fixing incorrectly-executed transactions (i.e., post-contract execution).

\footnotetext{
${ }^{10}$ For instance, a source of stock market pricing data could readily be compared to an alternative source.

${ }^{11}$ While such a device could give an inaccurate reading due to malfunction, this could be protected against with redundancy (i.e., polling multiple devices).
} 
New costs arising from secure execution. Blockchain technology facilitates the temporally-ordered processing and recording of transactions over time. This is enabled by a peerto-peer network of computers that process transactions as they occur, record these transactions in blocks of data, and enhance the blockchain's security by creating a theoretically immutable chain of data through continuous network consensus. Once consensus is reached, the peer-to-peer network updates and saves the decentralized database on several computers in real time to guarantee a transaction's security without the need for centralized intermediaries to ensure trust between transacting parties (Mougayar, 2016; Seidel, 2018 A). To illustrate, once network validators record a batch of transactions, aggregated data blocks are sealed and added to the data chain and a new block of data is then opened to record the next batch of transactions.

A prominent security risk to recording and executing blockchain-based transactions in this fashion is that of the 51-percent attack. This vulnerability refers to instances where a single nefarious entity (or bloc of entities) gains majority control of the network, thus enabling it to undo actual transactions and/or create false transactions. This occurs since reaching "consensus" in such cases depends on a single node or few colluding nodes rather than a large, distributed peer-to-peer network (Nesbitt, 2019). It follows that this security risk is heightened for lowernode networks. ${ }^{12}$ To address this security concern, organizations that depend on blockchainbased consensus may, in the future, incur costs for the services of entities that ensure a

\footnotetext{
12 While the number of Bitcoin network nodes exceeded 10,000 in March 2019 (Bitnodes, 2019), several blockchain networks are considerably smaller and thus more susceptible to this type of attack.
} 
blockchain's security by monitoring network concentration in real time and subsequently "deploying" their computing power when network concentration becomes too great. ${ }^{13}$

It is also important to recognize that the consensus-generating process is computationally intensive, and thus highly inefficient in terms of energy consumption when compared to centralized databases (Verkhivker, 2018). However, centralized databases are only as secure as the servers on which they reside. There are hardware, software, and labor costs with which a firm must contend to maintain a centralized database's security relative to blockchain technology. Given the prominence of large data breaches in recent years, one could argue that many firms are currently not spending enough to secure their servers. At the very least, caution is advised for scholars and firms alike when comparing the costs of blockchain security against the security expenses of traditional information technology.

\section{Implications of Blockchain for Contracting in the Market}

While spot market transactions are low risk in terms of whether and how they will be executed, many inter-firm agreements involve significantly greater levels of complexity (Schepker et al., 2014). The use of blockchain to develop smart contracts between firms is thus important for both theory and practice. Contracting in the market, often between firms, relies upon varying degrees of (1) identifying and knowing potential counterparties, (2) trust or assurance that counterparties can satisfy their performance obligations, and (3) ability to effectively monitor the counterparties (Dyer \& Singh, 1998; Gulati, 1995). Further, transactions can become more complicated when there is a time lag prior to fulfillment by one or both parties.

\footnotetext{
${ }^{13} \mathrm{We}$ are grateful to an anonymous reviewer for encouraging us to elaborate on 51-percent attack vulnerability in greater depth.
} 
As a result, intermediaries may be necessary to assist with monitoring and/or holding assets until a contract's prespecified conditions are met.

Blockchain technology is potentially impactful in addressing the various concerns that arise when firms contract between one another. Blockchain technology makes it easier to identify potential counterparties, reduces the amount of trust necessary between parties, and lessens the degree of monitoring that is required (Seidel, 2018 A). Rather than relying on an intermediary to address time lags or the transfer of assets, the technology itself can determine whether and when an exchange occurs in real time.

Since "a firm's capabilities influence the contracting structure it uses and the performance it realizes" (Schepker, 2014: 204), a potential implication of smart contracts is that they may impact power dynamics that are present in companies based on relative size (Josefy et al., 2015). Presently, larger counterparties may be able to exert influence over the favorable interpretation of a contract for which the smaller counterparty has little recourse. Even if the smaller party has a reasonable basis for pursuing legal action, it may be threatened or intimidated into dropping the claim because its pockets are not deep enough to hire sufficient legal resources to match those of the larger counterparty. Instead, if the contract is executed automatically in accordance with prespecified terms, the risk is substantially reduced for the smaller counterparty at the time it enters into a contract with the larger counterparty. Since scholars have also argued that inter-firm contracts are often executed with significant deviation from what is written, with the document existing primarily as a legal tool (Schepker et al., 2014), blockchain has the potential to help reduce relational dynamics and place greater emphasis on documented terms.

Following this reasoning, a key use case for blockchain-enabled smart contracts in existing firms is monitoring traditionally opaque supply chains. As mentioned above, both IBM 
and start-up Provenance have developed platforms to assist other firms in doing just that. Several large established firms such as Dow Chemical, DuPont, and Walmart are working with IBM Blockchain to improve their supply chains to increase the level of transparency for shareholders and other stakeholders who desire more information about sourcing practices. For instance, Walmart is piloting a blockchain solution to "dramatically improve traceability and transparency" of its food supply chain by digitally recording each step in a product's journey (i.e., where it is located, conditions of its location, what has been done to it, etc.) to create a realtime immutable record of a product's farming, harvesting, processing, and distribution (IBM, 2018). As such, blockchain-enabled supply chains reduce agent-managers' discretion over the portrayal of a firm's operations since a product's inputs are immutably recorded and transparent for any stakeholder to see, either by viewing a publicly-available distributed ledger in the case of a public blockchain or requesting and obtaining access to a distributed ledger (if not part of the authorized "consortium" of users) in the case of a private version.

\section{Implications of Blockchain for Contracting Within the Firm}

As outlined by agency theory, agent-managers may not always act in the best interest of a firm's owners or shareholders. This leads to agency costs both from self-serving agent behavior and from the costs incurred by the firm's efforts to prevent such behavior (Jensen \& Meckling, 1976). Some agency costs can be mitigated or even eliminated by blockchain technology, but others are beyond the scope of blockchain technology's influence. First, we examine the impact of blockchain on monitoring agent motivations and firm operations. Then, we discuss its lack of impact on excess expenses and interest alignment compensation. Finally, we turn our attention to blockchain's indirect mitigation of unrealized profits. 
Blockchain's impact on monitoring agent motivations. The 100-percent owner-manager intrinsically knows his or her own (owner's) interests. Yet, time, effort, and financial costs are associated with monitoring agent-managers to detect and deter self-serving behavior. Much of this monitoring focuses on the agent's motivations in executing and proposing changes to a firm's strategy. This monitoring, often focused on the CEO's actions and decisions, is typically carried out by the board of directors. Much corporate governance research has therefore focused on various characteristics of the board that can impact its (in)effectiveness of monitoring managers (Dalton et al., 2007; Walls, Berrone, \& Phan, 2012). Further research has considered the (in)dependence of directors on and from the CEO. While some researchers argue that boards are never truly independent (e.g., Davis \& Thompson, 1994; Galbraith, 2004), others argue that various measures of independence are still important for policing the $\mathrm{CEO}$ and improving firm profitability. For instance, directors appointed by the previous CEO are less likely to be beholden to the current CEO (e.g., Daily \& Dalton, 1995). Independence of the directors aside, annual surveys of corporate administrative assistants show that "corporate strategy discussions" as a key role of the board, grew from 31 percent in 2008 to 67 percent in 2012 (Spencer Stuart, 2012). Research supports the idea that shareholders with large holdings are better able to monitor agents than the typical minute fraction-of-outstanding-shares stockholder (e.g., Ang et al., 2000). Large stockholders selling off their shares in the firm can have a significant impact on share price and thus executives' wealth tied up in equity and options, making agent-managers more willing to accommodate them. Further, some large shareholders receive (or occasionally demand) seats on the board to allow them direct, rather than indirect, influence on the agent-managers.

One solution to the problem of opaque agent-manager motivations is to replace agentmanagers entirely. Blockchain technology creates the opportunity for owners to substitute smart 
contracts for agent-managers through a DAO organizational form. One challenge in completely replacing agent-management with smart contracts is that all contingencies must be accounted for in advance when creating them. As seen in the previous example of The DAO investment fund, the organization's inability to react to a hacker's unforeseen exploitation of its protocols led to its dissolution. As previously emphasized, this reflects the cost of inflexibility associated with smart contracts.

While full-fledged DAOs represent an extreme implementation of "managing" with smart contracts, it is also possible to envision how owners could use blockchain technology to execute complex routines repeatedly; therefore extending their ability to manage a firm by prolonging the time until agent-managers are necessary and/or limiting the functions of agent-managers. This is very much akin to how firms with greater technological capabilities also have more discretion in determining when to conduct transactions within or outside their boundaries (Mayer \& Salomon, 2006). Similarly, blockchain could provide owners with greater choice over when (and the degree to which) they need to rely on agent-managers.

The propensity for blockchain to eliminate (or substantially reduce) a firm's need to monitor agent-managers' motivations also brings forth several questions for researchers to consider. As evidenced by many instances where investors oust founder-CEOs in lieu of seasoned leaders with greater levels of experience (Wasserman, 2003), firms benefit from the leadership of individuals with considerable knowledge and expertise. Thus, further research can attend to the key junctures at which these "professional" managers are needed, and if the wisdom of decentralized crowds, combined with secure protocols, can eliminate the benefits gained by these leaders. Prior research also suggests early-stage ventures benefit from ambidexterity (Raisch, Birkinshaw, Probst, \& Tushman, 2009) and the ability to "continuously morph" in 
response to their dynamic environments (Rindova \& Kotha, 2001). Given this consideration, studies can attend to whether autonomous organizations are more or less flexible, and whether this form of governance has an optimal lifespan, after which certain functionalities are off-loaded to agent-managers based on specifications in the initial code. Researchers can also seek to identify the key junctures when responsibilities can be shifted from smart contracts to agentmanagers (and vice versa).

Taken together, blockchain-enabled smart contracts allow firms to eliminate agentmanagers in lieu of many direct owner-managers. However, once this governance structure is ingrained in a blockchain's protocols, it is difficult (if not impossible) to override later without encoding contingencies ex-ante. Firms must, therefore, consider many possible future scenarios, particularly low-probability rare events, that owner-managers may be ill-equipped to address when creating initial algorithmic smart contracts. This is consistent with the idea that ownermanagers are boundedly rational and thereby unlikely to be able to account for every possible rare event in the smart contracts' contingencies (Simon, 1957). Scholars can therefore attend to several normative questions such as: what types of decisions should be encoded in smart contracts, whether humans should be able to override a democratized and automated governance structure, and the processes by which established firms can effectively integrate blockchainenabled smart contracts into their existing firms to decentralize certain aspects of governance while maintaining control over others. This is further discussed in the next section on how blockchain can be used to monitor a firm's operations.

Blockchain's impact on monitoring firm operations. The second source of agency costs is the monitoring of a firm's operations due to concerns over information asymmetries. Managers have an informational advantage over shareholders and even directors about firm operations. 
Thus, managers may at times present a distorted, overly-optimistic picture of a firm's operations to shareholders and/or their intermediary, the board of directors. For this reason, some scholars have argued against the practice of recruiting directors from other industries-for the sake of independence—-since such directors lack the industry-specific knowledge necessary for effective monitoring (Dalton, 2005). This rosy view can be used, for instance, to justify commencing or continuing a non-profit-maximizing strategy. Stock and industry analysts, as well as large creditors, can also serve a role in reducing the information asymmetry of the agent-managers by bringing key metrics to light. Information asymmetry also presents agent-managers with an additional (non-profit-maximizing) incentive to expand a firm's operations into new product areas or industries to further enhance their advantage since the additional complexity reduces transparency and increases the uncertainty between actions and outcomes (Anderson, 1999; Brown \& Eisenhardt, 1997).

While restructuring extant organizations to run entirely on smart contracts is both impractical and infeasible for many existing firms, there are several areas where blockchain technology provides real benefits for such firms, particularly in monitoring day-to-day operations. As discussed above, owners must monitor a firm's operations since agent-managers have an information advantage over owners, shareholders, and directors regarding a firm's dayto-day operations. Though most agent-managers refrain from engaging in abject fraud, information asymmetries do allow agent-managers to over-optimistically present a firm's operations and/or conceal less-than-optimal circumstances. Thus, blockchain-enabled smart contracts offer a valuable way to monitor a firm's operations, and thereby mitigate the need for third-party intermediaries such as auditors to verify managers' claims, industry analysts to validate key firm metrics, and authenticators to monitor a firm's supply chain handoffs. 
Blockchain-enabled smart contracts are potentially an effective way for principal-owners to monitor aspects of a firm's operations, such as its supply chain, and thereby mitigate agentmanagers' discretion over certain day-to-day operational decisions. This would create value for shareholders in several ways. First, it enables a firm's managers to respond more accurately and rapidly to unforeseeable events (e.g., if and when to discard produce during a nationwide salmonella outbreak based on real-time supply chain data). This degree of information availability reduces agent-managers' discretion in determining a course of action and therefore mitigates the loss of value for shareholders due to managers' misinformed decisions and/or mishandled situations. Second, for products in which sourcing is important to customers, firms could provide consumers with access to objective information on the blockchain regarding a product, such as its embodied energy or its environmental footprint. This not only enhances end consumers' experience but also has the potential to reduce the costs associated with intermediaries by reducing the time and effort required to validate a product based on a variety of dimensions and considerations.

Though blockchain provides an effective means to monitor a firm's operations by reducing information asymmetries and mitigating the need for costly intermediaries to validate agent-managers' claims, blockchain does not replace an agent-manager's role in developing a firm's strategy. For example, blockchain-enabled supply chains may be able to certify whether a product is "organic" or "Fair Trade," but managers are still needed to determine whether it is advantageous for a firm to provide a product with this certification in the first place; unless, of course, decisions are completely decentralized as in the case of DAOs. This gives rise to an interesting consideration as to whether firms can utilize blockchain-enabled smart contracts to autonomously, or semi-autonomously, implement strategic decisions via algorithms that consider 
external variables to then trigger actions under certain circumstances. Further research can attend to whether and how firms construct smart contracts to make and implement strategic decisions and the performance implications of automated strategy-making.

Taken together, the use of blockchain to monitor firms' operations restricts an agentmanager's ability to make false claims or conceal information from shareholders. While this provides many obvious advantages to shareholders and other stakeholders including suppliers, exchange partners, and end consumers, it also introduces potential challenges and therefore new avenues for scholarly inquiry. One issue arises from the amount of transparency a firm introduces into its operational activities. Granting authorized users, such as exchange partners in a supply chain, access to immutable information reduces monitoring costs. Scholars can examine the circumstances under which firms benefit from public, permission-less blockchains that are completely transparent; private, permissioned blockchains that are restricted to select users; or consortium blockchains that blend together elements of the permissioned and permission-less blockchains (DeFranco, 2017). These disclosures may affect how consumers evaluate the products they buy or provide new inputs into the "market for classification" (Fleischer, 2009). Another question pertains to the role of the board of directors when a firm's operations are transparently recorded on a blockchain. While prior findings suggest boards are in place to monitor a firm's operations, if operational decisions are transparent, it remains to be seen how the role of the board will adapt and change over time. For example, when blockchain solutions are implemented to monitor a firm's operations, the board's composition may shift to comprise advisors from outside the focal industry because the need for novel and innovative ideas is more valued by a firm than operational expertise. 
Blockchain's impact on excess expenses. The third source of agency costs is that of “excessive" expenses by agent-managers, with excessive constituting the portion of an expense over and beyond what a 100-percent owner-manager would spend in their place (Ang et al., 2000; Jensen \& Meckling, 1976). Excessive expenses from managerial perks represent a potential agency problem "because the manager gains 100 percent of each dollar spent on perks, but only a percentage of each dollar in firm profit" (Ang et al., 2000: 84). This agency cost is typically more of a concern for smaller firms since the cost of lavish perks for executives can easily represent a higher percentage of a firm's revenue and profit ${ }^{14}$. For larger firms, the potential costs of executive perks, even the most extravagant, pales in comparison to the profits at risk from the firm's diversification or acquisition strategy. For large firms, one can argue that acquisitions to grow the size of the firm to obtain higher executive compensation fit the category of excessive expenses (Hoskisson et al., 1999; Tosi \& Gomez-Mejia, 1989), although this fits equally well under "suboptimal" management leading to unrealized profits as discussed below.

Unfortunately, blockchain technology is ineffective at mitigating excess expenses. As long as firms continue to transact and hold bank accounts in fiat currencies, blockchain holds little hope of reducing executive perk extravagance; thus, it provides no agency cost relief for smaller firms where excessive perks can amount to a significant portion of a firm's profit and revenues. Additionally, as discussed above, blockchain technology affords no new avenue to discover an agent's motivations. Unnecessary acquisitions and/or other similar excessive expenses are therefore still a potential problem for larger firms. However, this assertion makes one critical assumption: fiat currencies will remain the dominant means by which firms transact.

\footnotetext{
${ }^{14}$ It is worth noting that executives drawn to extravagant perks may incorporate them into their compensation contract during recruiting. This practice can effectively remove such costs from possible mitigation.
} 
If firms increasingly transact with cryptocurrencies, each transaction will be recorded on a blockchain's distributed ledger. As such, the degree to which a blockchain is public or private will determine the transparency of executives' expenses. While firms could utilize private blockchains wherein certain authorized personnel validate expenditures, this is not so different from systems currently in place in large firms; however, firms could also utilize public blockchains wherein transactions could potentially be visible to principal-owners outside the firm. If that occurs, the threat of scrutiny may serve as a check on executive spending. Further, the cost of monitoring executive spending would decrease, such that institutional investors, reporters, or even individual investors could choose to investigate expense patterns to check for potential waste or abuses. It remains to be seen how transacting in cryptocurrencies will impact agent-managers' excessive expenses.

Blockchain's impact on interest alignment compensation. The fourth source of agency costs consists of all the performance-based compensation (e.g., equity, stock options) a firm spends to better align agent-managers' interests with those of its owners. By compensating agent-managers, largely in equity, options, and other "incentive" pay based on stock price and/or profits, agents' interests are brought more in line with other shareholders. For instance, equitybased compensation reduces the temptation for extravagant executive perks since partial-owner agent-managers receive a portion of the additional profits when they chose to forego 100-percent perks as mentioned above (Ang et al., 2000). Further, the agents also have their employment at risk in their equity-based income and wealth. Still, research has shown that to effectively align agents' interests with those of owners, incentive compensation must represent a significant portion of the agent's overall wealth (Nyberg et al., 2010). This means that, with time, the cost of an agent-manager's incentive alignment will increase. 
While blockchain-enabled smart contracts provide several benefits over traditional contracts, on a fundamental level, they are still contracts. As such, a good contract is one that outlines all of the possible contingencies up front. In the case of employment contracts, this would mean spelling out the work to be performed, the expected quality of such work, and the compensation for said work. As smart contracts are essentially computer programs comprised of several rules and contingencies, the work output in employment contracts needs to be an objective measure that can be codified and then verified when it is time for a payment to be released. This presents the challenge of codifiability; specifically, it is far easier to codify the exact job duties of an entry-level employee than that of a high-level executive. For example, an account manager at Amazon has several responsibilities which include negotiating contracts, meeting sales quotas, and preparing reports on an account's status. Each of these tasks can be codified and compensation can be released in accordance with each task's successful execution. In contrast, a senior executive at Amazon has more ambiguous responsibilities such as largescale strategic decision-making, responding to unforeseeable problems, and mitigating sources of uncertainty in a firm's decision settings. These tasks are less codifiable and therefore harder to automatically compensate based on their successful execution.

Based on the above description, smart contracts fail in aligning the interests of agentmanagers with principal-owners via compensation for the same reasons a traditional contract largely fails at preventing shirking or other non-profit-maximizing behaviors (at least directly) amongst agent-managers; that being, the tasks of agent-managers are often too ambiguous to specify in contractual terms. However, smart contracts can sidestep this difficulty in the same way traditional contracts do so, by basing compensation on owners' desired outcomes (i.e., share price, profits, revenues, etc.) rather than agent-managers' actual strategic decisions and actions. 
While technically possible to implement via smart contracts, these high-level, performance-based executive compensation agreements are already readily and efficiently enforced through traditional contracts. Therefore, a smart contract seemingly offers no real benefit to principalowners from an agency cost perspective, particularly when the costs associated with coding such smart contracts in the first place are considered.

While blockchain-enabled smart contracts do not provide an effective or efficient means to align principal-owners' interests with those of agent-managers in established firms, it is worth noting that in DAOs, where agent-managers are removed entirely, compensation is determined by the smart contracts' protocols on which the organization is based. Returning to the example of The DAO, owner-managers determined how to allocate The DAO's pooled funds by voting on proposals. Payouts to owner-managers were then based on the funds they allocated to a proposed project and the project's subsequent achievement of key metrics specified within the smart contracts' protocols. In this case, the concept of "alignment" between principals and agents was rendered obsolete since agent-managers were replaced by owner-managers. Though interesting in terms of scalability and democratized decision-making, DAOs do not so much differ in terms of interest alignment compensation from other closely-held firms where owners have not delegated management and owners' returns are directly tied to their firms' performance.

Taken together, blockchain-enabled smart contracts are ill-suited to supplant regular employment contracts as a means to align principal and agent interests via compensation. While it is feasible to compensate lower-level employees via smart contracts since their responsibilities largely encompass clearly defined tasks, this does little to alleviate agency costs since these employees are not involved in firm-wide strategic decisions. However, when considering the nature of agent-managers' tasks, it is challenging for smart contracts to specify agent-managers' 
ambiguous responsibilities to align both parties' interests. Nevertheless, future studies could examine firms' efforts to codify agent-managers' compensation packages to understand if certain forms of smart employment contracts are better able to incentivize agents to align with principalowners' interests. Further, setting defined rules in advance could decrease the likelihood that performance benchmarks are revised post-hoc to later award managers unwarranted shares. For instance, smart contracts could have potentially avoided the options back-dating scandals that numerous firms perpetuated, or the selective revision to peer groups for firms to reach their desired performance benchmarks and therefore trigger the vesting of particular share awards.

Blockchain's impact on unrealized profits. The final source of agency costs are the unrealized profits that arise when agent-managers pursue personal rather than profit-maximizing goals. While the exact amount of unrealized profits is difficult to assess as it can even include missed opportunities, this is arguably the primary cost feared by owners since it often represents the largest potential agency cost (i.e., profits lost due to a suboptimal firm strategy can dwarf the expense of auditors or executive perks). However, due to the interrelated nature of the agency problem, this cost is largely the outcome of ineffective monitoring and interest misalignment. There are many ways an agent-manager can pursue a personally-driven suboptimal strategy for the firm rather than the profit-maximizing goal preferred by owners. For instance, the CEO could pursue a diversification strategy, especially unrelated diversification, that tends to reduce risk but also profitability (Hoskisson \& Turk, 1990). Assuming profitability does not fall too much, such a move provides the agent-manager with additional job security at the cost of lower profits for shareholders. Executives may also favor pursuing strategies to grow the size of the firm to obtain higher compensation rather than higher profits (Hoskisson et al., 1999; Tosi \& Gomez-Mejia, 1989). Further, increases in both diversification and firm size also lead to larger information 
asymmetry as the added complexity of large, diverse firms causes them to be more difficult to monitor than small, focused firms (Anderson, 1999; Brown \& Eisenhardt, 1997). Unrealized profits might also manifest in the form of stymied $R \& D$ expenditures or thwarted new product development if agent-managers believe their employment risk is better managed by avoiding mistakes rather than maximizing profits and taking sufficient risks. Finally, suboptimal returns for owners may be the result of shirking by the CEO, wherein CEOs do not expend maximum effort on enhancing employees' performance.

The cost of unrealized profits is largely the outcome of (in)effective agent-manager monitoring and interest alignment. As such, mitigating this agency cost is dependent on the extent to which blockchain is implemented within the firm. On one end of the spectrum, DAOs eliminate the agency problem entirely and therefore remove all agency costs, including unrealized profits, since all decisions are made by owner-managers via a democratic voting process and executed through network consensus. On the other side, for large existing firms implementing blockchain-based solutions in certain areas, the improved transparency reduces monitoring costs and the informational advantages of agent-managers. This should indirectly reduce unrealized profits to some extent. Finally, in the somewhat special case of ownermanagers extending the size of a firm with smart-contract "managed" employees, one could argue that the firm can achieve profits that exceed what "In the original Jensen and Meckling agency theory, the zero agency-cost base case is... the firm [managed and] owned solely by a single owner-manager" (Ang et al., 2000: 81).

In summation, we suggest blockchain technology can mitigate some agency costs. Specifically, blockchain has the potential to significantly impact the costs associated with monitoring agent motivations and firm operations and, indirectly, unrealized profits. However, 
blockchain technology holds less promise of relieving owners from the agency costs associated with excessive expenses and interest alignment compensation.

\section{FUTURE RESEARCH}

This manuscript explores how blockchain technology can (and is) impacting contracting costs both in the market and within organizations. In this paper, we first introduce blockchain technology and then discuss prior literature on contracting costs. We then discuss three distinct costs that result from contracting using blockchain technology. Next, in relation to contracting on the market, we discuss how blockchain impacts counterparties' willingness to transact with one another. Finally, in relation to contracting within the firm, we summarize five sources of agency costs from prior literature and address the ways blockchain technology is being (or can be) used to mitigate these five agency costs that firms have historically faced. In light of the implications of blockchain for contracting, we offer many avenues for future research.

First, there are several promising avenues for scholars to investigate the impact of blockchain technology on market transactions, including transaction costs, trust, and the role of intermediaries. Transaction costs have long influenced firms' decisions to contract in the market or internalize transactions. Certainly, technology has long played a role in changing the relative costs of types of transactions. Yet, as discussed, blockchain introduces new types of costs that must also be considered. Future research can address whether firms, and their managers, are adept at recognizing and assessing these costs and optimizing contracting decisions accordingly. In doing so, future research — both theoretical and empirical—can assess the impact of blockchain on expanding or contracting firm boundaries (Josefy et al., 2015; Zenger et al., 2011).

Similarly, blockchain may provide the impetus for new research on the importance of trust in contracting decisions. For instance, some have argued that the trust required for relying 
on blockchain in the marketplace shifts trust in the counterparty to trust in the overall system surrounding blockchain (Schneier, 2019). The willingness to rely on blockchain may vary in different ways than the willingness to rely on other counterparties or other ways of transacting.

This is related to another notion long considered central to studying market transactions: the role of intermediaries. Many transactions include at least one (or more) intermediary — banks, for instance, to clear payments. Indeed, some industries, such as real estate, rely on a complex set of intermediaries (Pollack, Porac \& Wade, 2004). As certain traditional intermediaries are displaced by blockchain — itself a new type of "intermediary" — scholars could consider how this alters willingness to conduct certain transactions or how it creates new vetting processes and routines. For instance, vetting data sources presents different challenges than vetting traditional intermediaries. Meanwhile, some organizations may seek to limit their exposure to any one public blockchain while others may seek potential efficiencies by conducting as many transactions as possible on the same underlying blockchain.

Second, we suggest several additional areas for scholars to investigate the impact of blockchain on corporate governance within organizations. We assert the continued need for agent-managers - even in organizations predominantly "managed" through smart contractsunder certain situations and circumstances. When rare events occur that are not (or cannot be) anticipated in the contingencies of an organization's preprogrammed smart contracts, we hold that humans are still necessary to orchestrate a pathway forward. This suggests that without a certain degree of human intervention, smart contracts alone may be ill-equipped to deal with reality as it unfolds in real time. However, the degree to which agent-managers have control over an organization's autonomous smart contracts is an area ripe for further inquiry. Future research can examine the organizational functions that most benefit from being automated by blockchain- 
enabled smart contracts. While it may make sense for an organization's day-to-day valuecreating operational decisions to be carried out via smart contracts, it is unclear what other organizational decisions should be preprogrammed into smart contracts.

For instance, in revisiting the example of The DAO, a centralized agent-manager was unavailable, and therefore powerless, to stop a hack as it occurred in real time or even put safeguards in place to prevent similar incidents in the future. As a result, The DAO was unable to recover from the hack and disbanded soon after its occurrence. While The DAO is an extreme example of a decentralized autonomous organization, its implications carry over to hierarchical organizations attempting to incorporate blockchain-enabled smart contracts. Future research can compare the impact of blockchain-enabled smart contracts in automating different aspects of an organization's decision-making environment on a firm's longer-term performance. Specifically, studies can consider the normative implications of using smart contracts to automate strategymaking, operations, and/or responses to environmental stimuli. This brings up two additional considerations: organizational ambidexterity versus rigidity, and the catalysts for agent-manager intervention in blockchain-based firms.

While smart contracts can effectively mitigate certain agency costs, they often limit an organization's ability to adapt. This may be particularly disadvantageous for early-stage entrepreneurial firms seeking to develop capabilities (Rindova \& Kotha, 2001), form organizational boundaries (Santos \& Eisenhardt, 2009), and gain initial power in exchange relationships (Ozcan \& Eisenhardt, 2009). For these firms, smart contracts may introduce an undesirable degree of rigidity that could subsequently hinder their ability to engage in strategyforming activities. Future research can thus examine emergent blockchain-based firms versus emergent hierarchical firms to better understand how reductions in human agency influence a 
firm's longer-term viability and success. In this vein, future research can also examine the junctures when organizations benefit most from implementing blockchain-enabled smart contracts. For instance, prior work on entrepreneurial firms' networking activities suggests firms have the most to gain from tie-forming activities with exchange partners after achieving uncertainty-reducing "proofpoints" (Hallen \& Eisenhardt, 2012). Similarly, there may exist "proofpoints" when firms are able to benefit most from implementing smart contracts. As such, the relationship between uncertainty (particularly the mitigation of uncertainty) and the use of smart contracts is an important area of further scholarly inquiry.

Another line of future inquiry can revisit the root of agency costs to further understand if and how principal-agent relationships are fundamentally different within blockchain-based firms. To illustrate, Jensen and Meckling (1976) discuss how agency problems arise because contracts have costs: both to be written and to be enforced. Given the autonomous nature of blockchainbased smart contacts, the costs to enforce such contracts are significantly reduced and/or eliminated (with the exception of miners' fees to reach consensus on transactions). However, it is important to consider that smart contracts may in some instances have a higher cost to write. Future research can examine the cost-benefit of monitoring agent-managers' behaviors via smart contracts versus drafting contracts in a traditional fashion. Specifically, a study recording the monitoring costs present in traditional organizations compared to blockchain-enabled firms (or even DAOs) for comparable functions would allow for a robust assessment of the magnitude of cost reduction. Testing the structure of blockchain-enabled firms in this way may reveal that the three types of costs we have outlined are greater than expected, that there are hidden costs in addition to those we have outlined, and/or there are other downsides that mitigate the positive effects of using smart contracts. 
Future research can also consider smart contracts as the "agent" for principal-owners. This will become increasingly important as smart contracts expand their interface with artificial intelligence to autonomously execute a variety of tasks such as purchasing materials, selling products, and halting shipments. In this way, technologies will increasingly create protocols and act on them with little (or no) human intervention. As such, the role of principal-owners in determining the initial contingencies of smart contracts is a critical consideration. This can also catalyze further research on how firms integrate the far-reaching objectives of various principalowners into their strategic decisions, beyond only profit-maximizing financial objectives, since social and environmental metrics can also be preprogrammed into a firm's smart contracts.

In addition to the direct impacts of blockchain technology on agency costs as discussed in this manuscript, blockchain technology may also have even further-reaching impacts on organizational dynamics. For instance, the digital tokens held by token holders in a blockchainbased firm often provide voting rights. Given the digital nature of such tokens, it can be assumed that token holders are digitally connected. This allows owners to interact and exercise their voting rights through online engagement rather than traditional stockholder meetings and/or physical proxy statements. This reduced voting burden may tilt blockchain organizations toward a direct democratic dynamic in lieu of the "representative government" that is typical of boards of directors. Whether such a shift toward more direct involvement of many small-share owners is good for a firm's performance (or other outcomes) is fertile ground for future research.

Blockchain-enabled firms also differ in how they deal with conflict resolution based on several structural characteristics. The form of governance that a firm adopts influences its structure (Provan \& Kenis, 2008). Different blockchain-enabled firms fall at different points along the continuum of governance concentration. For instance, the governance of Bitcoin is 
different from the governance of alternative public blockchains. This is directly determined by the protocols on which the blockchain network is based and the token distribution mechanics. First, protocols can favor different parties in a firm's decision-making process by granting voting rights on distinct criteria (e.g., number of tokens owned, reputation score, or level of prior engagement). Second, token distribution determines whether a firm's tokens (which grant voting rights) are all able to be publicly mined (i.e., Bitcoin) or pre-mined by the firm (i.e., Ripple, which pre-mined 50 billion of its 99 billion token units to ensure the firm's insiders maintained substantial voting power). Whether firms centralize governance, as in the case of traditional hierarchical firms using blockchain to monitor certain day-to-day operations, or share decentralized governance, as in the case of DAOs, this foundational difference impacts how conflicts can be resolved when they arise. This is an important consideration since a blockchainenabled firm that relies on multiple parties with differing interests will likely face more conflicts than those that are more reliant on a smaller set of entities to make decisions. The various uses of blockchain and their impact on conflict resolution in organizations is an important area for further inquiry.

Finally, another benefit afforded to token holders (i.e., owners) in blockchain-enabled firms is that the transparency they realize does not depend on their proportionate stake in the system. This is different than most traditional organizations where the representation of smaller interests is typically poor. When ownership concentration increases in such firms, conflicts of interest often arise between large and small shareholders, particularly when majority owners appoint management willing to comply with their interests (Shleifer \& Vishny, 1997). These large shareholders can then enrich themselves at the expense of smaller shareholders through related-party transactions and a variety of other mechanisms (Ho \& Wong, 2001). However, in 
blockchain-enabled firms, particularly in the extreme case of DAOs, smaller shareholders have more access to the operations of an organization. Smaller shareholders are thus able to ensure the code operating the system aligns with their interests. They are not cut out from scrutinizing how the organization operates and also have the ability to withdraw their proportionate stake if they feel their interests are not being represented (Jentzsch, 2016 B). In summary, these owners in blockchain-based firms know the code-base they monitor is the one in use because of the trust created by the underlying blockchain architecture. They also know the transparent protocols through which such firms are altered and the rights they possess to disconnect their financial stake from operations if they no longer believe a firm is operating in their interests.

\section{CONCLUSION}

This manuscript illustrates several ways blockchain-enabled smart contracts can impact firm contracting. Upwork, the largest global freelancing website, recently reported the fastestgrowing demand on its platform was for blockchain-skilled freelancers for two straight quarters

(Upwork, 2018). The growth in blockchain use cases suggests that it is increasingly important for scholars to consider how new technologies such as blockchain have the capacity to alter the way firms contract and even supplant human agency in organizational decision-making. While smart contracts have the capacity to mitigate certain agency costs, they may also expose principalowners to additional costs and risks. While we maintain blockchain-based solutions hold potential as an effective and efficient means to mitigate certain contracting and agency costs, we also encourage researchers and practitioners alike to further consider and critically examine the long-term ramifications of smart contracts and autonomous governance for organizations. 


\section{REFERENCES}

Ambler, P. 2018. From Zero to Crypto Billionaire in Under a Year: Meet the Founder of Binance. Forbes. Retrieved from https://www.forbes.com/sites/pamelaambler/ 2018/02/07/changpeng-zhao-binance-exchange-crypto-cryptocurrency/\#6192c57bleee.

Anderson, P. 1999. Complexity theory and organization science. Organization Science, 10: 216232.

Ang, J. S., Cole, R. A., Lin, J. W. 2000. Agency costs and ownership structure. The Journal of Finance, 55: 81-106.

Argyres, N., \& Mayer, K. J. 2007. Contract design as a firm capability: An integration of learning and transaction cost perspectives. Academy of Management Review, 32: 10601077.

Berle, A. A., \& Means, G. C. 1932. The modern corporation and private property. New York: MacMillan.

Bitnodes. 2019. Global Bitcoin nodes distribution. Retrieved from https://bitnodes.earn.com.

Bogost, I. 2017. Cryptocurrency might be a path to authoritarianism. The Atlantic. Retrieved from https://www.theatlantic.com/technology/archive/2017/05/blockchain-ofcommand/528543/.

Bowles, N. 2018. Everyone Is Getting Hilariously Rich and You're Not. The New York Times. Retrieved from https://www.nytimes.com/2018/01/13/style/bitcoin-millionaires.html.

Brown, S., \& Eisenhardt, K. M. 1997. The art of continuous change: Linking complexity theory and time-paced evolution in relentlessly shifting organizations. Administrative Science Quarterly, 42: 1-34. 
Buterin, V. 2013. Ethereum white paper. Retrieved from https://github.com/ethereum/wiki/ wiki/White-Paper.

Carpenter, M. A., \& Sanders, W. G. 2002. Top management team compensation: The missing link between CEO pay and firm performance?. Strategic Management Journal, 23: 367375.

Chavez-Dreyfuss, G. 2016. Virtual company may raise \$200 million, largest in crowdfunding. Reuters. Retrieved from https://www.reuters.com/article/us-blockchain-crowdfundingidUSKCN0Y82LI.

Christidis, K., \& Devetsikiotis, M. 2016. Blockchains and smart contracts for the internet of things. IEEE Access, 4: 2292-2303.

Coase, R. H. 1937. The Nature of the Firm. Economica, 4.16: 386-405.

Columbus, L. 2018. IoT Market Predicted To Double By 2021, Reaching \$520B. Forbes. Retrieved from https://www.forbes.com/sites/louiscolumbus/2018/08/16/iot-marketpredicted-to-double-by-2021-reaching-520b/\#517371881f94.

Conlon, E. J., \& Parks, J. M. 1990. Effects of monitoring and tradition on compensation arrangements: An experiment with principal-agent dyads. Academy of Management Journal, 3: 603-622.

Cyert, R. M., \& March, J. G. 1963. A behavioral theory of the firm. Englewood Cliffs, NJ: Prentice-Hall.

Daily, C. M., \& Dalton, D. R. 1995. CEO and director turnover in failing firms: An illusion of change?. Strategic Management Journal, 16: 393-400.

Daily, C. M., Dalton, D. R., \& Cannella Jr., A. A. 2003. Corporate governance: Decades of dialogue and data. Academy of Management Review, 28: 371-382. 
Daily, C. M., Dalton, D. R., \& Rajagopalan, N. 2003. Governance through ownership: Centuries of practice, decades of research. Academy of Management Journal, 46: 151-158.

Dalton, D. R. 2005. "Going private" and "going dark;" board compensation, and the downside of independence. Directors \& Boards, 2: 4-6.

Dalton, D. R., Hitt, M. A., Certo, S. T., \& Dalton, C. M. 2007. The fundamental agency problem and its mitigation: Independence, equity, and the market for corporate control. The Academy of Management Annals, 1: 1-64.

Davis, G. F., \& Thompson, T. A. 1994. A social movement perspective on corporate control. Administrative Science Quarterly, 39: 141-173.

De Mol, L. 2018. Turing Machines, The Stanford Encyclopedia of Philosophy (Winter 2018 Edition), Edward N. Zalta (ed.). Retrieved from https://plato.stanford.edu/archives/ win2018/entries/turing-machine.

DeFranco, L. 2017. What would a blockchain expense policy look like? Retrieved from https://blog.abacus.com/blockchain-expense-policy/.

Dempsey, T., \& Conway, R. S. 1918. The Delphic Oracle: Its Early History, Influence and Fall. BH Blackwell: London.

Dyer, J. H., \& Singh, H. 1998. The relational view: Cooperative strategy and sources of interorganizational competitive advantage. Academy of Management Review, 23: 660679.

Eisenhardt, K. M. 1989. Agency theory: An assessment and review. Academy of Management Review, 14: 57-74.

Fama, E. F. 1980. Agency problems and the theory of the firm. Journal of Political Economy, 88: 288-307. 
Fama, E. F., \& Jensen, M. C. 1983. Agency problems and residual claims. The Journal of Law and Economics, 26: 327-349.

Feldman, M. S. 2000. Organizational routines as a source of continuous change. Organization Science, 11: 611-629.

Feldman, M. S., \& Pentland, B. T. 2003. Reconceptualizing organizational routines as a source of flexibility and change. Administrative Science Quarterly, 48: 94-118.

Fleischer, A. 2009. Ambiguity and the equity of rating systems: United States brokerage firms, 1995-2000. Administrative Science Quarterly, 54: 555-574.

Galbraith, J. K. 2004. The economics of innocent fraud. Boston: Houghton Mifflin.

Grant, R. M. 1996. Toward a knowledge-based theory of the firm. Strategic Management Journal, 17: 109-122.

Guadiano A.M. 2018. Most ICOs are unjustified, but soaring due to FOMO. MarketWatch. Retrieved from https://www.marketwatch.com/story/most-icos-are-unjustified-butsoaring-due-to-fomo-2018-01-23.

Gulati, R. 1995. Does familiarity breed trust? The implications of repeated ties for contractual choice in alliances. Academy of Management Journal, 38: 85-112.

Gupta, V. 2017. The promise of blockchain is a world without middlemen. Harvard Business Review. Retrieved from https://hbr.org/2017/03/the-promise-of-blockchain-is-a-worldwithout-middlemen.

Hallen, B. L., \& Eisenhardt, K. M. 2012. Catalyzing strategies and efficient tie formation: How entrepreneurial firms obtain investment ties. Academy of Management Journal, 55: 35 70. 
Ho, S. S., \& Wong, K. S. 2001. A study of the relationship between corporate governance structures and the extent of voluntary disclosure. Journal of International Accounting, Auditing and Taxation, 10: 139-156.

Hoskisson, R. E., \& Turk, T. A. 1990. Corporate restructuring: Governance and control limits of the internal capital market. Academy of Management Review, 15: 459-477.

Hoskisson, R. E., Wan, W. P., Yiu, D., \& Hitt, M. A. 1999. Theory and research in strategic management: Swings of a pendulum. Journal of Management, 25: 417-456.

Howard-Grenville, J. A. 2005. The persistence of flexible organizational routines: The role of agency and organizational context. Organization Science, 16: 618-636.

IBM. 2018. Retrieved from https://www.ibm.com/blockchain/platform.

Jensen, M. C., \& Meckling, W. 1976. Theory of the firm: Managerial behavior, agency costs, and ownership structure. Journal of Financial Economics, 3: 305-360.

Jentzsch, C. 2016 (A). Charity DAO. Retrieved from https://medium.com/charitydao/charitydao-e9592dd80ab7.

Jentzsch, C. 2016 (B). Decentralized Autonomous Organization to Automate Governance. Slock.it. Retrieved from https://download.slock.it/public/DAO/WhitePaper.pdf.

Josefy, M., Kuban, S., Ireland, R. D., \& Hitt, M. A. 2015. All things great and small: Organizational size, boundaries of the firm, and a changing environment. The Academy of Management Annals, 9: 715-802.

Kaplan, S. N., \& Stromberg, P. 2001. Venture capitals as principals: Contracting, screening, and monitoring. American Economic Review, 91: 426-430. 
Mahdawi, A. 2018. Meet Erik Finman, the teenage bitcoin millionaire. The Guardian. Retrieved from https://www.theguardian.com/technology/2018/jun/13/meet-erik-finman-theteenage-bitcoin-millionaire.

Mayer, K. J., \& Salomon, R. M. 2006. Capabilities, contractual hazards, and governance: Integrating resource-based and transaction cost perspectives. Academy of Management Journal, 49: 942-959.

Michelman, P. 2017. Seeing beyond the blockchain hype. MIT Sloan Management Review, 58: $17-19$

Mougayar, W. 2016. The business blockchain: Promise, practice, and application of the next internet technology. Chicago: Wiley.

Nakamoto, S. 2008. Bitcoin: A peer-to-peer electronic cash system. Retrieved from https://bitcoin.org/bitcoin.pdf.

Nelson, R. R., \& Winter, S. 1982. An evolutionary theory of economic change. Cambridge, MA: Belknap Press/Harvard University Press.

Nesbitt, M. 2019. Deep chain reorganization detected on Ethereum Classic (ETC). Retrieved from https://blog.coinbase.com/ethereum-classic-etc-is-currently-being-51-attacked33be13ce32de.

Northern Trust. 2017. Northern Trust and IBM pioneer use of blockchain technology to help transform private equity administration. Retrieved from https://www.northerntrust.com/ about-us/news/press-release?c=70b5ba1adc9928f9977162844c34f57a.

Nyberg, A. J., Fulmer, I. S., Gerhart, B., \& Carpenter, M. A. 2010. Agency theory revisited: CEO return and shareholder interest alignment. Academy of Management Journal, 53: 1029-1049. 
Ozcan, P., \& Eisenhardt, K. M. 2009. Origin of alliance portfolios: Entrepreneurs, network strategies, and firm performance. Academy of Management Journal, 52: 246-279.

Parmigiani, A., \& Howard-Grenville, J. A. 2011. Routines revisited: Exploring the capabilities and practice perspectives. The Academy of Management Annals, 5: 413-453.

Pollock, T. G., Porac, J. F., \& Wade, J. B. 2004 Constructing deal networks: Brokers as network "architects" in the US IPO market and other examples. Academy of Management Review, 29: 50-72.

Provan, K. G., \& Kenis, P. 2008. Modes of network governance: Structure, management, and effectiveness. Journal of Public Administration Research and Theory, 18: 229-252.

Raisch, S., Birkinshaw, J., Probst, G., \& Tushman, M. L. 2009. Organizational ambidexterity: Balancing exploitation and exploration for sustained performance. Organization Science, 20: 685-695.

Rindova, V. P., \& Kotha, S. 2001. Continuous “morphing”: Competing through dynamic capabilities, form, and function. Academy of Management Journal, 44: 1263-1280.

Roberts, J. J. 2018. Cryptocurrency scams are now among the SEC's top enforcement priorities. Fortune. Retrieved from http://fortune.com/2018/11/02/sec-ico-report-cryptocurrencyscams/.

Santos, F. M., \& Eisenhardt, K. M. 2009. Constructing markets and shaping boundaries: Entrepreneurial power in nascent fields. Academy of Management Journal, 52: 643-671.

Schepker, D. J., Oh, W. Y., Martynov, A., \& Poppo, L. 2014. The many futures of contracts: Moving beyond structure and safeguarding to coordination and adaptation. Journal of Management, 40: 193-225. 
Schneier, B. 2019. There's no good reason to trust blockchain technology. Wired. Retrieved from https://www.wired.com/story/theres-no-good-reason-to-trust-blockchaintechnology/.

Seidel, M-D. L., 2018 (A). Questioning centralized organizations in a time of distributed trust. Journal of Management Inquiry, 27: 40-44.

Seidel, M-D. L., 2018 (B). "Discussant Comments," Academy of Management Conference: Chicago, IL, Session 1250: Digital Ledger Technology: How Blockchain Is Changing Organizations and Markets, August 13.

Senta, L. 2017. Where and how application data is stored in Ethereum. Retrieved from https://www.singulargarden.com/blog/storage-and-dapps-on-ethereum-blockchain/.

Shleifer, A., \& Vishny, R. W. 1997. A survey of corporate governance. The Journal of Finance. 52: 737-783.

Simon, H. A. 1957. Models of man: Social and rational. Oxford, UK: Wiley.

Simon, H. A. 1977. The organization of complex systems. In Models of discovery (pp. 245-261). Springer, Dordrecht.

Sklaroff, J. M. 2018. Smart contracts and the cost of inflexibility. Retrieved from http://clsbluesky.law.columbia.edu/2018/01/04/smart-contracts-and-the-cost-ofinflexibility/.

Spencer Stuart. 2012. 2012 Spencer Stuart Board Index. Retrieved from http://womensleadershipfoundation.org/wp-content/uploads/2012/05/Spencer-Stuart-USBoard-Index-2012.

Stout, L. 2002. Bad and Not-So-Bad Arguments for Shareholder Primacy. Southern California Law Review, 75: 1189-1209. 
Tapscott, D. \& Tapscott, A. 2017. How blockchain will change organizations. MIT Sloan Management Review. 58: 10-13.

Thompson, J. D. 1967. Organizations in action. New York: McGraw-Hill.

Tosi, H. L., \& Gomez-Mejia, L. R. 1989. The decoupling of CEO pay and performance: An agency theory perspective. Administrative Science Quarterly, 34: 169-189.

Tosi, H. L., Katz, J. P., \& Gomez-Mejia, L. R. 1997. Disaggregating the agency contract: The effects of monitoring, incentive alignment, and term in office on agent decision making. Academy of Management Journal, 40: 584-602.

Upwork. 2018 Upwork releases Q2 2018 Skills Index, ranking the 20 fastest-growing skills for freelancers. Retrieved from https://www.upwork.com/press/2018/07/31/q2-2018-skillsindex.

Verkhivker, A. 2018. Why Bitcoin and blockchain may stumble. Chicago Booth Review. Retrieved from http://review.chicagobooth.edu/economics/2018/article/why-bitcoin-andblockchain-may-stumble.

Walls, J. L., Berrone, P., \& Phan, P. H. 2012. Corporate governance and environmental performance: Is there really a link? Strategic Management Journal, 33: 885-913.

Wasserman, N. 2003. Founder-CEO succession and the paradox of entrepreneurial success. Organization Science, 14: 149-172.

Weber, L., \& Mayer, K. J. 2011. Designing effective contracts: Exploring the influence of framing and expectations. Academy of Management Review, 36: 53-75.

Weber, L., \& Mayer, K. J. 2014. Transaction cost economics and the cognitive perspective: Investigating the sources and governance of interpretive uncertainty. Academy of Management Review, 39: 344-363. 
White, M. 2018 Digitizing global trade with Maersk and IBM. Retrieved from https://www.ibm.com/blogs/blockchain/2018/01/digitizing-global-trade-maersk-ibm/.

Williamson, O. E. 1979. Transaction-cost economics: The governance of contractual relations. The Journal of Law and Economics, 22: 233-261.

Williamson, O. E. 1981. The economics of organization: The transaction cost approach. American Journal of Sociology, 87: 548-577.

Williamson, O. E. 1985. The economic institutions of capitalism: Firms, markets, relational contracting. New York: Free Press.

Zenger, T. R., Felin, T., \& Bigelow, L. 2011. Theories of the firm-market boundary. Academy of Management Annals, 5: 89-133. 


\begin{abstract}
AUTHOR BIOGRPAPHIES
Alex Murray is an Assistant Professor of Management at the Lundquist College of Business at the University of Oregon. His research examines how emergent technologies impact the ways in which organizations and entrepreneurs mobilize resources, enroll stakeholders, and coordinate activities. He received his Ph.D. from the University of Washington.

Scott Kuban is an Assistant Professor of Strategy at the Freeman School of Business at Tulane University. His research examines CEOs, executives, and boards in areas including scandals, political activities, and social capital, and how technology is altering the nature of firms. He received his $\mathrm{Ph} . \mathrm{D}$. from Texas A\&M University.

Matthew Josefy is an Assistant Professor of Strategy and Entrepreneurship at the Kelley School of Business at Indiana University. His research examines changes in the fundamental nature of organizing and how these changes alter how organizations attract and manage resources, particularly human capital, how organizations are governed, and how firms engage with stakeholders. He received his Ph.D. from Texas A\&M University.

Jon Anderson is an Equity Options Trader at CMT Asset Management in Chicago. He completed his B.S. in Accounting and Finance at Indiana University where he was recognized with the Provost's Award for Undergraduate Research.
\end{abstract}

